Article

\title{
An Approach to Decompose and Evaluate a Complex GIS-Application Design to a Simple, Lightweight, User-Centered App-Based Design Using User Experience Evaluation
}

\author{
David Kammerhofer (D) and Johannes Scholz *(D) \\ Research Group Geoinformation, Institute of Geodesy, Graz University of Technology, 8010 Graz, Austria; \\ david.kammerhofer@student.tugraz.at \\ * Correspondence: johannes.scholz@tugraz.at
}

Received: 27 July 2020; Accepted: 20 August 2020; Published: 23 August 2020

\begin{abstract}
Working with contemporary, monolithic desktop Geographical Information Systems can be laborious and confusing, especially for non-experts. An alternative for a more user friendly approach to spatial data edition and spatial analysis may be an "app-based" web application that is offering single tasks for the user- "micro applications". In this paper, a method for determining such "micro applications" based on user stories is presented and applied to a specific use case- the case is centered around an Austrian Governmental Institution that digitizes and edits infrastructure data. The results of this process are implemented in an app-based web application. To measure the impacts of the app-based approach, we evaluate the user experience of the app-based approach in comparison to a desktop Geographical Information System offering the same functionality. For the measurement of the user experience, we used a focus group. The group had to accomplish tasks with the desktop Geographical Information System and with the app-based web application. To measure their user experience, we employed the tool AttrakDiff. The app-based web application achieves significantly better results in terms of user experience. This result is confirmed in a discussion panel carried out afterwards. Test persons responded that positive aspects of the app-based web application were the easy and intuitive handling and reduced user interface that helps the users to focus on their tasks without any distraction.
\end{abstract}

Keywords: user experience evaluation; app-centered approach; web application; GIS; user-centered design

\section{Introduction}

A Geographical Information System (GIS) is a system for capturing, storing, editing, management, analysis, and visualization of spatial data [1,2]. A classical environment for GIS is the desktop computer. GISs that are installed as desktop programs gained high popularity among users during the 1980s and 1990s because they could be used from any PC and could be customized accordingly. Due to the increased functionality of desktop GIS, there are traditional barriers of desktop GIS [3,4]. In particular, for occasional or novice users, full-fledged desktop GIS are far from being intuitive pieces of software. Nevertheless, any occasional users in organizations may have to fulfill complex tasks concerning spatial data collection, editing, or analysis. In recent decades, the term GIS represents even more than a desktop software or a single system. The term serves as an umbrella term for a number of techniques and methodologies [5] in different application contexts. Spatial data analysis may be performed without a desktop GIS software, just by using a programming language and specific libraries (e.g., Python or R) [6,7] or fully online using a client-server based architecture [8]. In addition, the field of 
CyberGIS combines cyberinfrastructure, e-science, and GIS. CyberGIS focuses on computational and data-intensive geospatial problem-solving within various research and application domains $[9,10]$.

This paper is intended to contribute to decompose an existing and established workflow to process spatial data sets implemented as an extension to a commercial desktop GIS. The objective of the decomposition process is to have different "micro applications" tailored to fulfill a specific task. These "micro applications" can be designed in a way that reflects the user requirements at most. Selected closely related "micro applications" are subsequently combined with single web-based applications-denoted as the app-based approach. The research question is if an app-based approach shows a different User Experience (UX) than a desktop GIS approach for a set of defined spatial data editing and analysis functionalities. UX is a term that describes the feeling of a user when interacting with a specific product or service [11]. We intend to compare the UX-with the help of the tool AttrakDiff-of the app-based approach with the contemporary desktop GIS application and evaluate the results accordingly.

The approach is tested at an Austrian Institution that is collecting spatial data in the field of infrastructure. The selected institution utilizes a commercial desktop GIS approach to perform their complex tasks. On top of the desktop GIS, a number of specific tools have been implemented and deployed. However, the user interface is completely overcrowded from a visual point of view. Hence, users may be distracted quite easily. Furthermore, unnecessary input windows, input fields or similar complicated intuitive work and make it difficult for non-experts to work on a single task. In the paper, we use a focus group to evaluate the app-based approach and the desktop GIS approach of the Austrian Institution.

In Section 2, we briefly review the state of the art in the fields UX and user centered design in GIS. Section 3 explains the methodology of the decomposition process to end with a number of simpler web-based apps. In addition, we elaborate on the basics of user experience. In Section 4, the process of UX measurement is demonstrated. Section 5 summarizes the results, and Section 6 discusses the result and lists some further research in this field.

\section{Related Work}

The related work section lists related work in the field of user experience, cartographic interaction, interactive maps, and user-centered design. There have been numerous works in the field of map interaction design and interactive maps, and user experience in general, but only a handful of user experience evaluations for the purpose of designing web-based GI systems/apps have been published so far.

Interface design has the goal to create "usable" interfaces that ensure a seamless communication between an application and the user. Early works in this arena books by Medyckyj-Scott and Hearnshaw [12] and Nyerges et al. [13] (with an introductory chapter on cognitive aspects of HCI and GIS [14]). Still valid is the finding of Gould [15] that GIS projects may fail due to the ignorance of user requirements. In order to focus interface design on the user centered design approaches [16], user experience evaluation has been developed. The term UX is described by Hassenzahl [17] as a "momentary, primarily evaluative feeling (good-bad) while interacting with a product or a service". In addition, Hassenzahl [17] refers to good UX as the "consequence of fulfilling the human needs for autonomy, competency, stimulation, relatedness, and popularity through interacting with the product and service". The measurement of UX is done with several methods that are explained in e.g., [18,19].

Contemporary publications in the field of interactive map design focus on usability. As mentioned above, there are numerous works that deal with the usability evaluation of interactive maps (e.g., [20-23]). Schnur et al. [24] look at the visual complexity of popular online map applications like Google Maps or Bing Maps. In order to evaluate the usability of interactive maps, eye-tracking can be employed in combination with "classical" usability evaluation-see, e.g., [25]. In literature, there are some recent works that use UX methodologies in the context of Geographic Information Systems (e.g., [26-28]). In addition, the application of user experience methodologies to web-based 
maps and mapping applications has been mentioned by [22]. Roth [21] also concluded that some more work on the intersection of UX and and online mapping is worth researching. Roth [29] as well as Kray et al. [30] agree on the fact that there is nothing like a general and consolidated path to design map interactions. Degbello et al. [31] assume that this is due to the fact that usability evaluations and studies are very much dependent on the specific product one is evaluating. Gestalt principles have an influence on the placement of and arrangement of elements and their perception-which can be of interest for the map design, according to Traun [32]. The papers of Atzl [33,34] deal with general design principles and guidelines for map design and map-oriented dashboards for different user roles in particular.

Cartographic interactions have been defined by [35] as an interaction between a map and a human being. The interaction is manifested as a question that is subsequently answered with the help of a map. Roth [36] defined a task-given to a user-that consists of a combination of goal, objective, and operand. Roth [36] specified each term, goal, objective, and operand in a taxonomy, which was found valuable by Degbelo and Kauppinen [37]. Nevertheless, [37] concluded that the taxonomy should be improved for the purpose of web mapping.

According to Roth [21], user-centered design "describes the process of ensuring interface success-map-based or otherwise - by gathering input and feedback from target users throughout the design and development of the interface". Roth [21] also claims that a successful interactive map in practice has three components: the user, its utility, and its usability, which interact with each other in a loop. For Brehl [38], the focus of software development is on the goals and needs of the system's end users. It is also proposed to combine the advantages of user-centered design and agile software development to design a useful and usable software. Cellina et al. [39] applied the user-centered design approach to attempt to affect behaviour change in the field of individual mobility.

\section{Methodology}

In this section, the methodology of the decomposition process of the desktop GIS application is described. We elaborate on the determination of micro applications and the combination of related micro apps for the end user. In addition, we highlight selected applied user experience models.

\subsection{Overall Methodology and Approach}

The overall methodology followed in this paper is depicted in Figure 1. Based on the contemporary (existing) workflow(s), user stories are created. These user stories are intended to decompose the complex workflows into atomic tasks or micro applications. These micro applications are then analyzed concerning their characteristics. Based on the characteristics of each micro application, they are combined to single applications that are implemented.

To evaluate the app-based approach before fully implementing it, we intend to perform the UX testing with the help of a high-fidelity prototype [40,41] —a so called click dummy. According to Lim et al. [42], such prototypes are filters for exploring the design to support finding a final decision and may serve as a manifestation of a design idea.

In order to compare the UX of the app-based approach with the contemporary desktop GIS approach, we select a number of tasks that should be performed with both approaches. The UX evaluation is done using the AttrakDiff tool. A focus group was asked to perform the selected tasks using the app-based and the desktop-GIS approach and answering the questions of the AttrakDiff tool. 


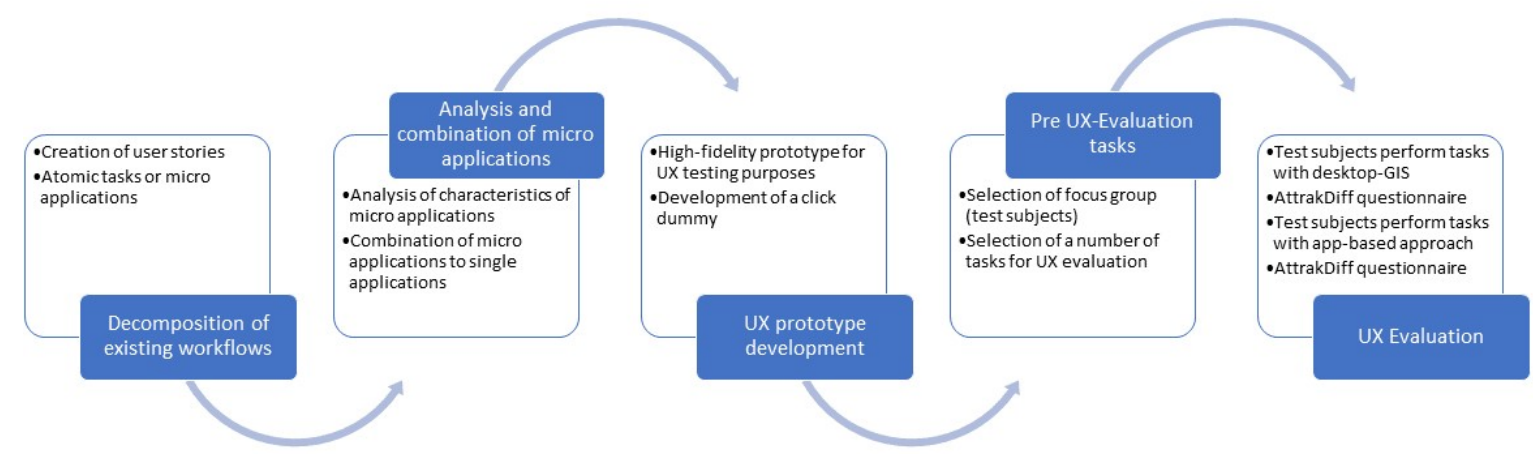

Figure 1. The overall workflow followed in the paper. The overarching methodological pillars are in the blue rectangles, whereas the finer grained methodological pieces are given in the white rectangles.

\subsection{Determination of Applications}

The aim is to disassemble complex workflows and processes in a way that enables us to determine the functionality of apps, which can be implemented in an app-based GIS web application. The approach in this paper uses elements from AGILE software development [43].

Similar to AGILE software development, the methodology proposed here collects the workflows and their representation as user stories. A user story in AGILE software development represents an informal description of one (or more) features of a software system in natural language. In this context, user stories serve as the basis for determining the micro applications. A user story describes who the editor is, the target of a workflow and the result. Furthermore, all requirements are listed. In Figure 2, an example of the structure of user stories is depicted.

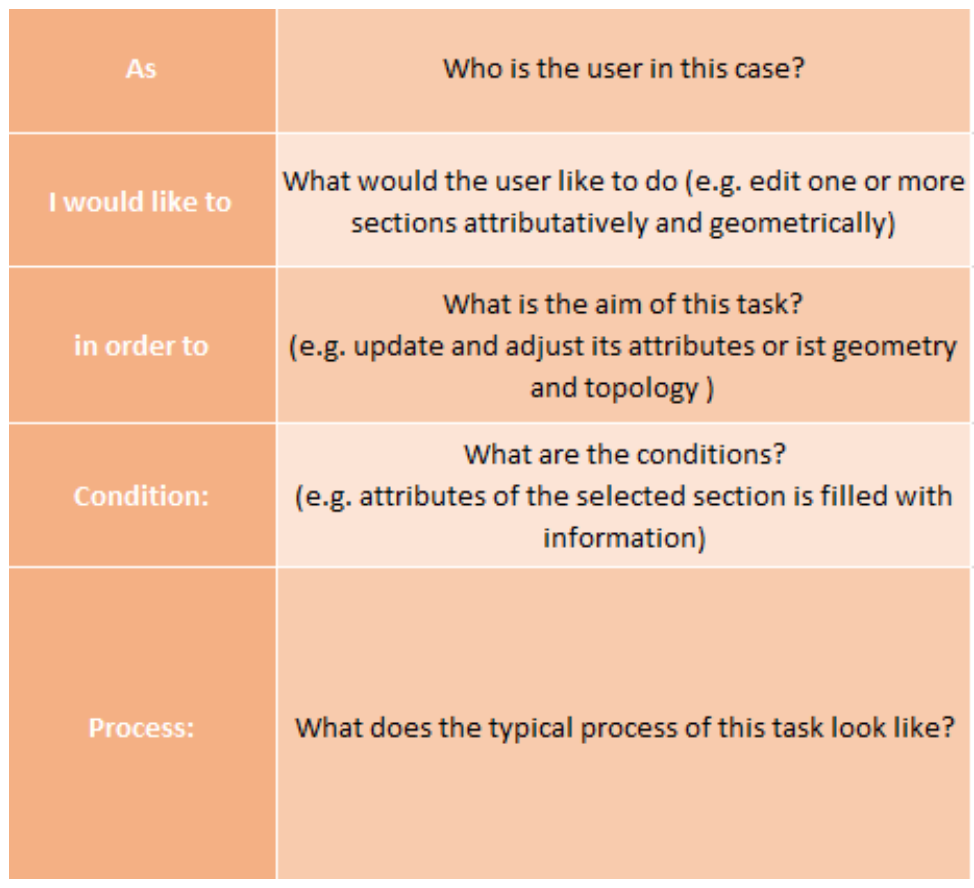

Figure 2. The structure of user stories applied in the experiment.

At the beginning of the app decomposition process, all user stories are defined as so-called micro applications. A micro application is an application that does not represent a single application because it is assumed that the functionalities are too small and too simple. However, several micro apps can be combined to form a single app. In addition, we identified basic components of applications-where components denote basic functions of applications and do not have to be reloaded in a new browser 
page (e.g., selections, routing). All micro applications are then examined concerning their potential to be combined with other micro apps with respect to the following characteristics:

- type of input data

- quantity of input data

- data manipulation characteristics (i.e., which specific type of spatial data editing/analysis function is required)

- contextual data type

- contextual data quantity

- type of output data

- quantity of output data

- components of the micro application

Subsequently, micro applications are combined into single applications. This is done on the basis of the relationship mentioned above. A relationship between micro applications is regarded to be strong if they have the same type of input data. For example, micro apps are combined that relate to sections of infrastructure data (edit sections, delete sections, and create sections). In addition, the data quantity of input, context, and output data are necessary to determine the feasibility of combining several micro applications. The objective of this is, to avoid apps with large amounts of data to be processed which might result in performance issues. If the amount of data to be processed in an application becomes so big that a web browser can not handle this in terms of a fluent user friendly performance, the application must be "divided" so the data can be processed in a web-based environment. This process can therefore be considered as iterative.

\subsection{User Experience Evaluation}

One perspective on user experience is the hedonic/pragmatic model of UX. This model assumes that people perceive interactive products along two different dimensions. The pragmatic quality (PQ) describes the perceived ability, a product can support achieving "do-goals". In other words, the pragmatic quality considers what a user does with a product and therefore reflects a concrete benefit such as by "making a telephone call" or "setting-up a web page". In contrast, the hedonic quality (HQ) describes the perceived ability, a product can support achieving "be-goals", such as "being competent" or "being special". In other words, the hedonic quality refers to what products symbolize or what new possibilities a product offers [11].

$\mathrm{PQ}$ and $\mathrm{HQ}$ are considered as different, independent dimensions of a product. A product designer consciously or unconsciously combines both qualities. For example, a layout is designed clear (PQ) and with an attractive color selection (HQ) at the same time. Both qualities are used in the assessment of a product's attractiveness. According to this model, the attractiveness is considered as an independent dimension and as an overall rating. PQ and HQ contribute to a product's attractiveness in equal parts. Hence, a low pragmatic quality can be balanced by a high hedonic quality and vice versa [11].

To measure the UX of the app-based approach in comparison to the desktop GIS approach, the AttrakDiff tool is used [44]. According to the developers of AttrakDiff, this tool offers the ability to measure UX-related product perceptions and assessments. Therefore, this tool applies the hedonic/pragmatic model of UX mentioned above. The usage of this research and evaluation tool is free of charge.

We have chosen this tool due to its easy and intuitive handling. Furthermore, AttrakDiff offers fast and well visualized illustrations of results of comparisons of different products in terms of the user experience. This tool offers three different types of projects (Single Evaluation, Comparison A-B and Before-After). AttrakDiff can be considered as a semantic differential. It is therefore a procedure to find out which ideas the tested people associate with specific terms. Only indirect questions are asked, which gives the tested person the opportunity to tell how strongly they connect a term with certain 
properties. A total of 28 bipolar (opposite) terms are queried, whereby the test person can choose from seven levels [45].

These 28 terms are combined into four scales, whereby each scale can be derived from seven terms. These four scales are [45]:

- Pragmatic quality: This is the perceived ability of a product to achieve "do-goals" by providing useful and usable functions. For example, if a pen has the utility of being able to write on a sheet of paper, it is sufficiently pragmatic for a user if this pen can actually write on a sheet of paper. For this pragmatic perspective, it is not important what this pen looks like, it is only important that the goal (writing on a sheet of paper) is achieved. Appropriate attributes for this are: practical, predictable, and clearly structured.

- Hedonic quality-stimulation (HQ-S): HQ-S describes the ability of a product to meet the need to improve one's own knowledge and skills. The product is intended to promote the personal growth of the user. Appropriate attributes for this are: creative, challenging, and inventive.

- Hedonic quality-Identity (HQ-I): This explains the ability of a product to communicate a self-serving message to others. This explains how a user of a product identifies himself with the product in a social context and what message the user wants to communicate to others. An example of this is a pen made of wood instead of plastic. A user who is very committed to sustainability identifies himself with a pen made of sustainable materials and also wants to show his efforts towards sustainability to the outside world. Suitable attributes for this are: brings me closer to people, professional, and connective.

- Attractiveness (ATT): This is the global positive-negative evaluation of the product. The user evaluates the product as a whole and describes it in simple words as good, appealing, or pleasant.

The survey data determined by the tested persons are automatically evaluated by AttrakDiff. The results essentially consist of three graphical representations (portfolio-presentation, diagram of average values, and description of word-pairs). The portfolio summarizes the results of pragmatic and hedonic (average values from identity and stimulation) quality. Furthermore, confidence intervals are determined, displayed as rectangles. This confidence rectangle indicates that a further study under the same conditions achieves a mean pragmatic respectively hedonic quality value inside this rectangle with a probability of 95 percent [45].

The goal of the UX measurement is to measure the effects on the user experience of dividing the complex work processes into apps and the implementation of these in an app-based web application. For the two project types in which products are compared, these confidence rectangles can be used to determine whether the difference in $\mathrm{HQ}$ or $\mathrm{PQ}$ is statistically significant (if they do not overlap). The larger the confidence rectangle, the more disagreeable the test participants were in the assessment. If the confidence rectangle is completely in a portfolio field (e.g., neutral or desired), the product can be assigned to a product character. In the diagram of average values, the mean values of the four scales (PQ, HQ-S, HQ-I and ATT) are depicted. In contrast to the portfolio-presentation, the hedonic quality is differentiated into the aspects of stimulation and identity. Furthermore, the attractiveness of a product is depicted. In the description of word-pairs, the mean values of the 28 individual word pairs are shown. The extreme values (those properties that are particularly critical or well solved) can be considered [45].

\section{Experiment}

The contextual setting of the experiment is as follows. In an Austrian Governmental institution, infrastructure-related data are collected, digitized, edited, and stored, to provide a seamless infrastructure dataset covering the area of Austria. In particular, each administrative body-e.g., community, province-is required to contribute and has to provide infrastructure-related data for the Austrian Governmental institution. The contemporary solution comprises of a commercial desktop GIS approach that is amended with a customized add-on application to the desktop GIS. 
Currently, the Governmental organization is thinking of providing a more user-centered solution for the administrative bodies that contribute infrastructure data. Thus, we intend to apply the methodology developed in Section 3 to the comparison of the contemporary desktop GIS vs. an application centered approach.

The app determination process shown in Section 3.2 is applied to 35 user stories. The following is an example of a description of characteristics of a user story. The example of creating a section is used for this. Furthermore, a combination of micro applications is shown.

At the beginning of this procedure, creating a section is regarded as an own micro application. However, there is no further information if this micro application will be combined with other micro applications to an app. The type of the input data are sections in the form of edges. Sections are usually digitized on a large scale because accuracy is essential, so the quantity of input data are low (only a few sections have to be shown in the web application, hence there are no performance issues). Data manipulation takes place when a new section is created.

To create a new section, additional contextual information like an appropriate basemap and further additional layers like borders are necessary. The output data type are also sections in a low amount of data (the user usually digitize just a few sections at once). Additional components of this micro applications are, among others:

- $\quad$ Snapping of edges to simplify digitizing

- Search sections by attributes (in the case the user looks for a special section)

- Show details of sections (e.g., name of the street) by mouse over for more than one second

This micro application is combined, among others, with the user story of edit sections. This user story's beginning also has its own micro application. This one has the same input data type (sections), quantity of input data (several sections), contextual data type and quantity (a basemap and additional layers like border), type and quantity of output data (also a few sections), and the same components. Only the data manipulation characteristics differ compared to the create section micro application (here the manipulation is to edit already existing sections). Hence, the relationship is considered to be strong and both micro applications are combined into one app. Further micro applications can then be added to this app.

To measure the UX changes between the desktop GIS and the app-based web application, the web-based application has to be developed first. In order to avoid a full implementation, we only developed a high-fidelity prototype (a click dummy). The prototype was developed based on the results of the app determination process (see Section 3.2). The prototypical application (i.e., click dummy) consists of a dashboard (see Figure 3) and several apps. With this app-based web application, attention is paid to a simple and clear design that is reduced to the bare minimum (see Figure 4). In order to be able to ensure that a user-centered design, there was an intensive exchange with a selection of people from the future user group. There was a weekly meeting to discuss current progress. The future user group suggested improvements that were taken into account in the implementation. In addition, switching between apps should be easy and intuitive. The spatial data are visualized with the library Leaflet [46] —an open source Java Script map library—and processed using a PostGIS spatial database [47]. The app-based web application prototype has the following properties: 


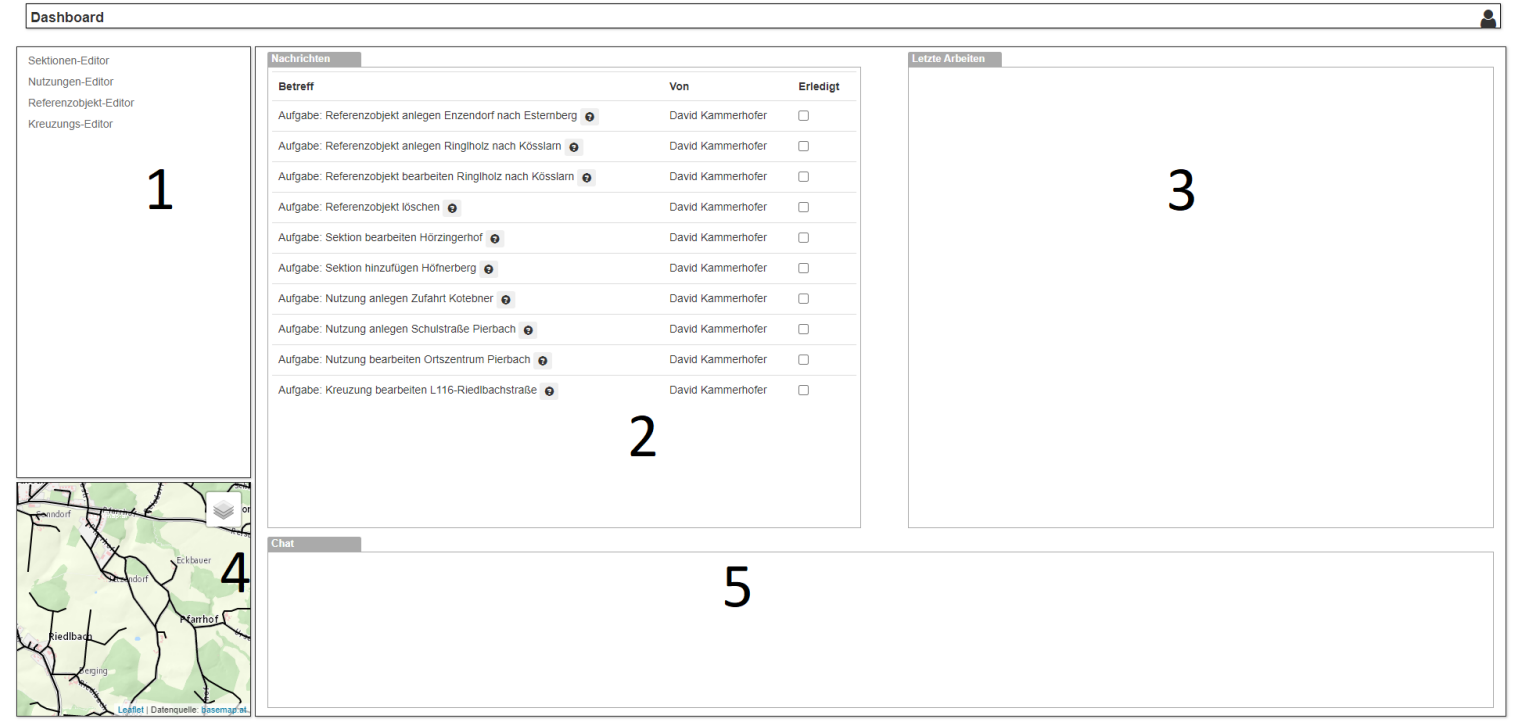

Figure 3. The implemented dashboard of the web application. It shows the list of links to the other apps (1); the list of tasks of the experiment with the link to pop-ups for further information (2); the suggestion of an implementation of a list of completed tasks (3); a small overview map (4); the suggestion of an implementation of a chat function (5).

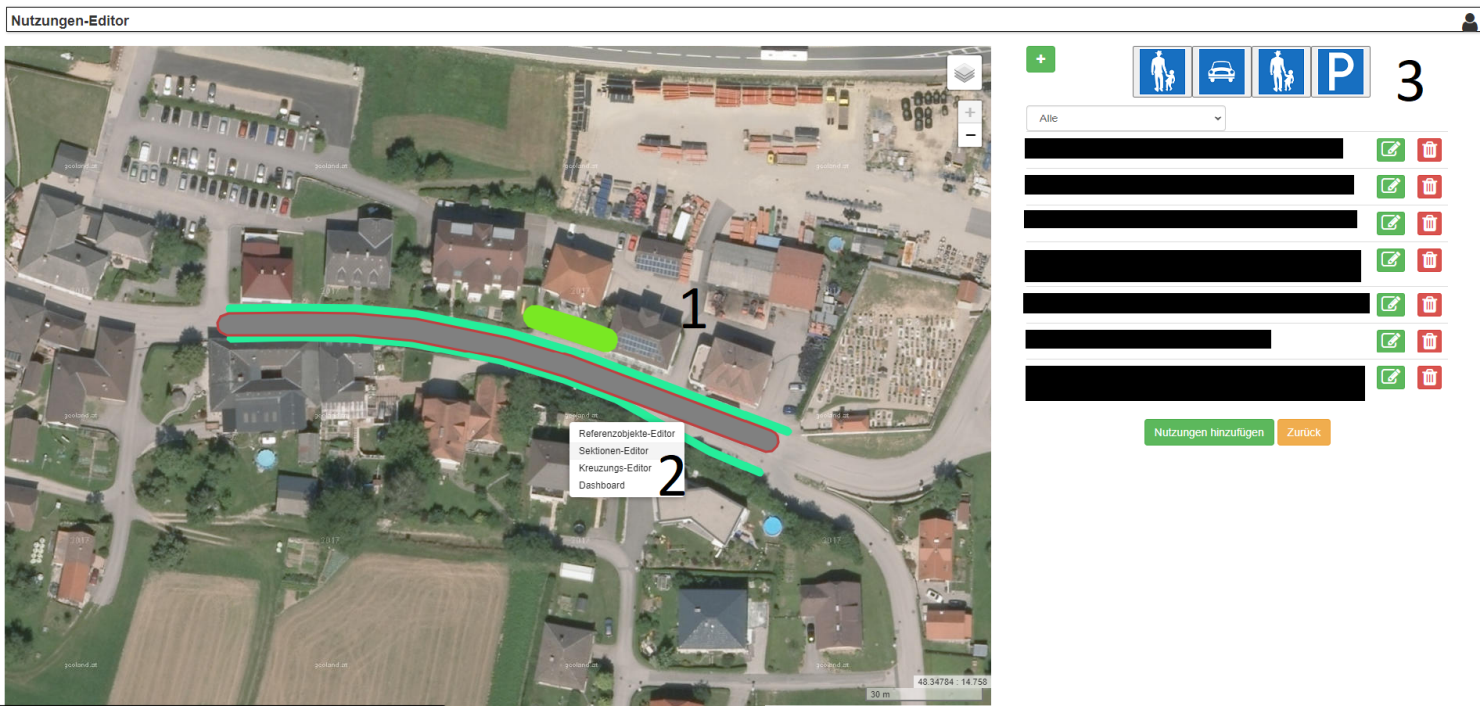

Figure 4. One of the implemented apps of the web application. It is showing the illustration of geodata (1); the easy and intuitive possibility to switch between different apps via context menu (2); an the schematic illustration of data with buttons and pictograms (the information is blackened due to confidentiality reasons) (3).

- $\quad$ Task bar: The task bar informs the user in which app they are currently working.

- Adjustment of basemap: The user is able to adjust the basemap quickly and easily. Therefore, there is the possibility to choose between three basemaps.

- Context menu: With any right click in an app on the map, a context menu should open. In this menu, links to other apps are displayed to ensure the user is able to switch between different apps quickly and easily. With a right click direct on geodata, an extended context menu opens, which, in addition to the links to the other apps, also shows special selection options. 
- Changing the URL: The URL indicates the position on the map (longitude and latitude from the center of the maps) as well as the zoom level. Every time the map view is changed, the URL updates accordingly.

- Passing parameters: If apps are called, the parameters in the URL are also passed at any time. By opening another app from the context menu, the parameters' longitude, latitude, and the zoom level are also sent per URL. The newly opened app uses these parameters to open the map at the same position and the same zoom level as the previously used app. Thus, the user is able to switch between apps smoothly. With the special selection options in the context menu (by right-clicking on an object), further parameters such as the object ID are also passed. This means that the corresponding object can be selected directly in the newly opened app and is directly editable.

To measure UX, a focus group with 14 participants was set up. This group consists of five women and nine men between the ages of approximately 25 and 60. Most of them have an academic education in the field of GIS. The others have at least several years of experience working with GIS. Thus, all of them are experts in working with the contemporary desktop GIS solution, especially working with infrastructure data. They work for several infrastructure companies and federal states and are part of the Austrian Governmental institution in the field of the development process and digitizing data. The goal of this focus group was to accomplish selected tasks first with the desktop GIS approach and afterwards with the app-based web application. We selected a number of 10 tasks that should be performed with the desktop GIS approach and the app-based approach. These selected tasks consisted of editing, creating, and deleting data. After processing, these tasks with the desktop GIS, the participants were asked to answer the questions of the AttrakDiff tool. Subsequently, after a short break, the focus group obtained a brief introduction working with the app-based web application, before processing the selected tasks again (this time with the app-based web application). After that, the participants of the focus group were asked to answer the AttrakDiff questions again. After completing the experiment, there was a discussion panel to exchange opinions and experiences.

\section{Results}

In the application determination process, 35 user stories were created-that were equal to micro applications. By applying the methodology highlighted in Section 3.2, we identified four applications, based on the 35 user stories. Together with the users, we called those applications "editor apps", which have the functionality to create, delete, and edit different kinds of spatial objects.

Those micro apps combined in one app only differ in the manipulation characteristic. Input data type, the quantity of the input data and the context are homogeneous within the apps and have therefore been combined. An exception to this is an app which, according to the methods above, was initially viewed as two separate apps. During the process of determining the apps, there was an intensive exchange with the future user group as they were able to incorporate their expertise. Hence, two editor apps have been combined to an app because users usually perform the workflows in these two apps at the same time.

The results of the UX evaluation are presented in the following paragraphs. Figure 5 depicts the results of the portfolio-presentation. The app-based web application (orange rectangle) performs better than the desktop GIS (blue rectangle) in terms of pragmatic (PQ) and hedonic quality (HQ). Both confidence intervals are pretty similar in size, which means the participants of the focus group are similarly agreed in terms of pragmatic and hedonic quality of both approaches (desktop GIS and app-based web application). Moreover, both confidence intervals do not overlap either horizontally or vertically. Therefore, the better results of the app-based web application in terms of pragmatic and hedonic quality are statistically significant. Hence, another study with the same test conditions has a 95 percent probability that the web application performs better than the desktop GIS in terms of $P Q$ and HQ again. In summary, the app-based web application is-for the participants of the focus group-better suited to achieve a specific goal. Moreover, the app-based approach shows better results 
in terms of the perceived quality and the feeling of "what this product has to offer", in comparison to the desktop GIS approach.

The diagram of average values (Figure 6) depicts the average values of the dimensions pragmatic quality, hedonic quality (this is divided into hedonic quality-stimulation and hedonic quality-identity), and attractiveness. These results show that the app-based web applications achieve better results in all dimensions-which is clearly to be seen by the orange line in Figure 6. This means a better perceived ability of the product (the web application) to achieve "do-goals" (pragmatic quality). Furthermore, the web application tends to satisfy the need to improve one's own knowledge (hedonic quality-stimulation) and the identification of the user with the application (hedonic quality-identity). In addition, users see the web application as more attractive and find the product better, more appealing and more pleasant (see Section 3.3).

Figure 7 shows the results of the description of word-pairs, which depicts all scores of the 28 different word-pairs. In this figure, the better results of the app-based web application in terms of UX are obvious. Of particular interest is the better performance of the web application in almost all word pairs (except unprofessional-professional). It is also notable that the app-based web application is in the positive UX range for all word pairs, except undemanding, challenging (here the score is exactly by zero). The desktop GIS approach, on the other hand, only manages to have two scores (unprofessional-professional and cheap—-premium) in the positive UX range.

\section{Portfolio-presentation}

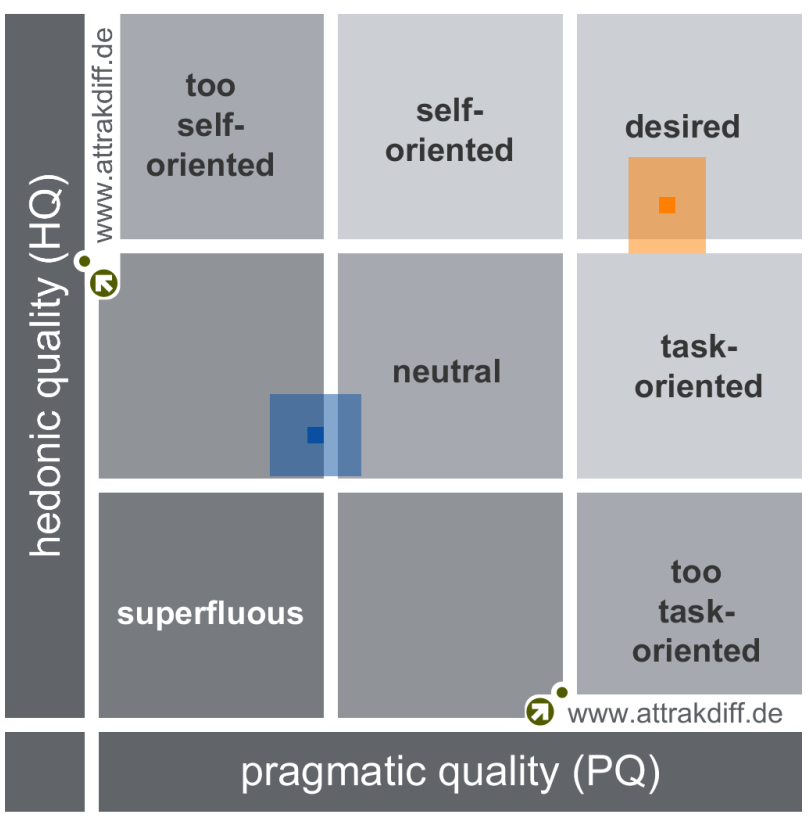

- Product: Desktop GIS $(n=14)$

$P Q:-1,16$ Confidence: 0,38

$\mathrm{HQ}:-0,58$ Confidence: 0,34

- Product: App based web application $(n=14)$

$P Q: 1,85$ Confidence:0,32

$\mathrm{HQ}: 1,38$ Confidence: 0,40

Figure 5. Result of UX-Measurement. (Portfolio-presentation). 


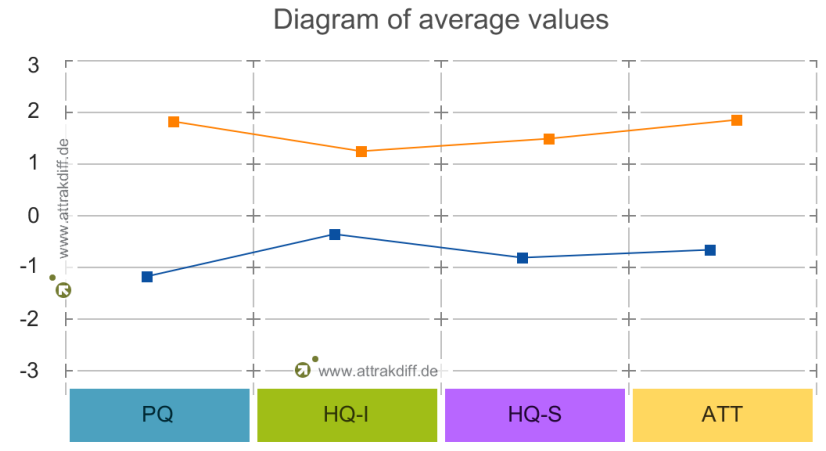

-Product: Desktop GIS ( $n=14)$

PQ:-1,16 HQ-I:-0,36 HQ-S:-0,81 ATT:-0,65

--Product: App based web application $(\mathrm{n}=14)$

PQ:1,85 HQ-I:1,27 HQ-S:1,50 ATT:1,86

Figure 6. Result of UX-Measurement (Diagram of average values).

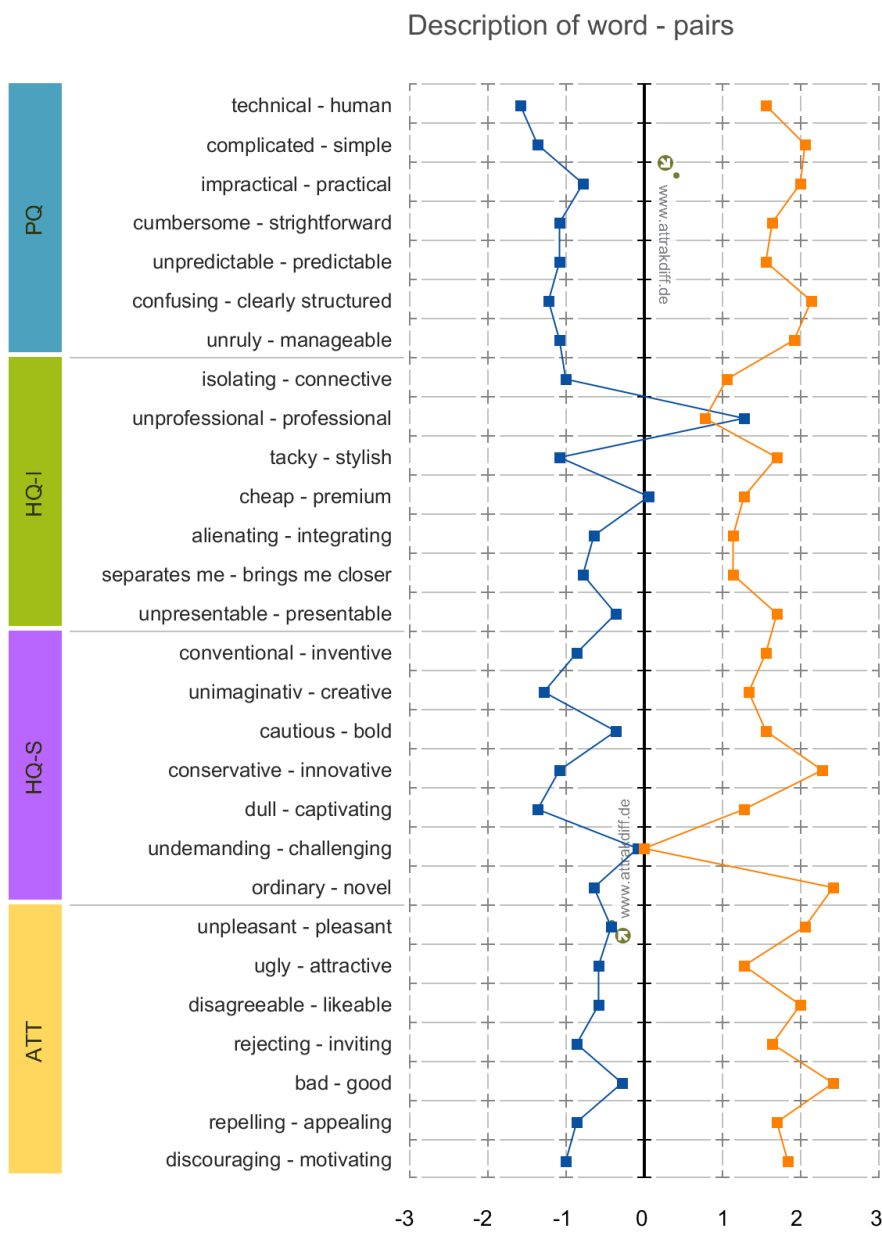

-Product: Desktop GIS ( $n=14)$

-- Product: App based web application $(n=14)$

Figure 7. Result of UX-Measurement (Description of word-pairs). 


\section{Discussion and Outlook}

With respect to the achieved results, working with an app-based GIS web application was more enjoyable for the focus group in terms of UX. The desktop GIS solution is regarded to be less intuitive from a user's point of view. This can be seen in the result of the user experience evaluation. The app-based web application is better suited to achieve a specific goal (pragmatic quality) and achieve better results in terms of the perceived quality of what this product has to offer. Moreover, the results show that the focus group perceives the app-based application to be, among others, more human, simpler, more innovative, and more clearly structured.

This result was also confirmed in the discussion panel within the focus group. The positive aspects mentioned most regarding working with the app-based web application were:

- $\quad$ easy and intuitive handling

- reduced user interface (no visual clutter or distractions)

Working with this web application can thus be quickly explained to non-experts due to its intuitive handling. In terms of working with the desktop GIS, the terms "laborious" and "confusing" were often mentioned by the focus group. This feeling is also confirmed in the results of the user experience evaluation. The desktop GIS is considered as more complicated and discouraging and also as more confusing compared to the app-based web application. This is where the advantage of the possibility of an individual, customer-specific implementation of an app-based web application comes into play.

The possibility of dividing the processes into applications in an app-based web approach can ensure that only the necessary, indispensable information (which is required for the respective processing of a specific task) is presented to the user. Furthermore, a somewhat schematic visualization of, for example, buttons is possible in a web application, which can make the handling of the web application easier, especially for non-experts. This leads to users seeing the product as more attractive, which the results clearly reflect as well.

To achieve a user-centered design, a periodic consultation with the future user group is required to identify design failures in an early stage (AGILE procedure). Thus, an attractive design with a good usability can be achieved. The future user of the application with its tasks, targets, knowledge, and skills is in the focus of the development process. The procedure should therefore be regarded as iterative, which can go through several feedback loops.

As mentioned above, an app-based web application has advantages regarding the user experience compared to a desktop GIS. If a company or an institution decides to migrate from a desktop GIS to an app-based web application, we recommend to consider a few things. First of all, all existing workflows have to be described as user stories. These user stories must be described using the characteristics of Section 3.2. Then, it can be examined which user stories have a strong relationship so that they can be combined. The results of the app determination should always be exchanged with the future user group. They often have a lot of experience and knowledge about special use cases.

When implementing the apps in an app-based web application, attention should always be paid to a simple and intuitive user-centered design. Here, it is also important to be exchanged with the future user group. If a prototype of an application is available, a UX measurement can be carried out. The AttrakDiff tool is well suitable for this. If a good result is achieved with this measurement, the technical implementation can be continued.

The focus of this work was on an application whose main task was editing, deleting, and creating infrastructure features-i.e., spatial data. The research question of whether the UX of the app-based approach is better in comparison to the desktop GIS approach can be clearly answered in light of the results. The UX evaluation of both approaches using a focus group clearly shows the advantages of the user-centered app-based approach.

Future works can be considered different types of an app-based web application's main tasks. Furthermore, the amount of data that can be processed in a web application can be determined in 
a future work. These results can be incorporated in the process of merging micro apps into single applications.

Author Contributions: D.K. and J.S. developed the research methodology. D.K. implemented the experiment (user stories and click dummy development and UX evaluation the focus group) and analyzed the results according. D.K. and J.S. wrote the initial manuscript, and revised the manuscript according to the suggestions of the reviewers. Both authors have read and agreed to the published version of the manuscript.

Funding: This research received no external funding.

Conflicts of Interest: The authors declare no conflict of interest.

\section{References}

1. Burrough, P.A.; McDonnell, R. Principles of Geographical Information Systems; Oxford University Press: New York, NY, USA, 1998.

2. Worboys, M.F.; Duckham, M. GIS: A Computing Perspective; CRC Press: Boca Raton, FL, USA, 2004.

3. Baker, T.R. Internet-based GIS mapping in support of K-12 education. Prof. Geogr. 2005, 57, 44-50.

4. Câmara, G.; Souza, R.d.; Monteiro, M.; Paiva, J.; Garrido, J.; Câmara, G.; Cartaxo, R.; Souza, M.; Miguel, A.; Monteiro, V.; et al. Handling complexity in GIS interface design. In Proceedings of the I Brazilian Workshop on GeoInformatics, Campinas, Brazil, 10-13 October 1999.

5. Maliene, V.; Grigonis, V.; Palevičius, V.; Griffiths, S. Geographic information system: Old principles with new capabilities. Urban Des. Int. 2011, 16, 1-6. [CrossRef]

6. Diener, M. Python Geospatial Analysis Cookbook; Packt Publishing Ltd.: Birmingham, UK, 2015.

7. Bivand, R.S.; Pebesma, E.J.; Gómez-Rubio, V.; Pebesma, E.J. Applied Spatial Data Analysis with R; Springer: Berlin/Heidelberg, Germany, 2008; Volume 747248717.

8. Dragićević, S. The potential of Web-based GIS. J. Geogr. Syst. 2004, 6, 79-81. [CrossRef]

9. Wang, S. A CyberGIS framework for the synthesis of cyberinfrastructure, GIS, and spatial analysis. Ann. Assoc. Am. Geogr. 2010, 100, 535-557. [CrossRef]

10. Wang, S. CyberGIS: Blueprint for integrated and scalable geospatial software ecosystems. Int. J. Geogr. Inf. Sci. 2013, 27, 2119-2121. [CrossRef]

11. Hassenzahl, M. The hedonic/pragmatic model of user experience. Towards UX Manif. 2007, 10, 10-14.

12. Medyckyj-Scott, D.; Hearnshaw, H.M. Human Factors in Geographical Information Systems; Halsted Press: Canberra, Australia, 1993.

13. Nyerges, T.L.; Mark, D.M.; Laurini, R.; Egenhofer, M.J. (Eds.) Cognitive Aspects of Human-Computer Interaction for Geographic Information Systems; Springer: Dordrecht, The Netherlands, 1995. [CrossRef]

14. Nyerges, T.L.; Mark, D.M.; Laurini, R.; Egenhofer, M. Cognitive aspects of HCI for GIS: An introduction. In Cognitive Aspects of Human-Computer Interaction for Geographic Information Systems; Springer: Berlin/Heidelberg, Germany, 1995; pp. 1-8.

15. Gould, M.D. GIS design: A hermeneutic view. Photogramm. Eng. Remote Sens. 1994, 60, 1105-1116.

16. Saffer, D. Designing for Interaction: Creating Innovative Applications and Devices; New Riders Pearson Education Distributor: Berkeley, CA, USA; London, UK, 2010.

17. Hassenzahl, M. User experience (UX): Towards an experiential perspective on product quality. In Proceedings of the 20th International Conference on Association Francophone d'Interaction Homme-Machine, IHM '08, Metz, France, 2-5 September 2008; ACM International Conference Proceeding Series; Brangier, E., Michel, G., Bastien, J.M.C., Carbonell, N., Eds.; ACM: New York, NY, USA, 2008; pp. 11-15. [CrossRef]

18. Goodman, E.; Kuniavsky, M.; Moed, A. Observing the User Experience: A Practitioner's Guide to User Research; Elsevier: Amsterdam, The Netherlands, 2012.

19. Albert, W.; Tullis, T. Measuring the User Experience: Collecting, Analyzing, and Presenting Usability Metrics; Elsevier: Newnes, Australia, 2013.

20. Andrienko, N.; Andrienko, G.; Voss, H.; Bernardo, F.; Hipolito, J.; Kretchmer, U. Testing the Usability of Interactive Maps in CommonGIS. Cartogr. Geogr. Inf. Sci. 2002, 29, 325-342. [CrossRef]

21. Roth, R.; Ross, K.; MacEachren, A. User-Centered Design for Interactive Maps: A Case Study in Crime Analysis. ISPRS Int. J. Geo Inf. 2015, 4, 262-301. [CrossRef] 
22. Poplin, A.; Guan, W.; Lewis, B. Online Survey of Heterogeneous Users and Their Usage of the Interactive Mapping Platform WorldMap. Cartogr. J. 2016, 54, 214-232. [CrossRef]

23. Morgan, J.D. A User-centered Design for the Addition of Interactive Masking Capability within an existing Web GIS. Trans. GIS 2016, 20, 807-816. [CrossRef]

24. Schnur, S.; Bektaş, K.; Çöltekin, A. Measured and perceived visual complexity: A comparative study among three online map providers. Cartogr. Geogr. Inf. Sci. 2017, 45, 238-254. [CrossRef]

25. Çöltekin, A.; Heil, B.; Garlandini, S.; Fabrikant, S.I. Evaluating the Effectiveness of Interactive Map Interface Designs: A Case Study Integrating Usability Metrics with Eye-Movement Analysis. Cartogr. Geogr. Inf. Sci. 2009, 36, 5-17. [CrossRef]

26. Hennig, S.; Vogler, R. User-Centred Map Applications Through Participatory Design: Experiences Gained During the 'YouthMap 5020' Project. Cartogr. J. 2016, 53, 213-229. [CrossRef]

27. Hodza, P. Evaluating User Experience of Experiential GIS. Trans. GIS 2009, 13, 503-525. [CrossRef]

28. Resch, B.; Zimmer, B. User Experience Design in Professional Map-Based Geo-Portals. ISPRS Int. J. Geo Inf. 2013, 2, 1015-1037. [CrossRef]

29. Roth, R.E. Interactive maps: What we know and what we need to know. J. Spat. Inf. Sci. 2013, 2013, 59-115. [CrossRef]

30. Kray, C.; Schmid, F.; Fritze, H. Guest editorial: Map interaction. GeoInformatica 2017, 21, 573-576. [CrossRef]

31. Degbelo, A.; Kruse, J.; Pfeiffer, M. Interactive maps, productivity and user experience: A user study in the e-mobility domain. Trans. GIS 2019, 23, 1352-1373. [CrossRef]

32. Traun, C. Wahrnehmungsorientierte Kartengestalung. In Online-Karten im Fokus: Praxisorientierte Entwicklung und Umsetzung; Henning, S., Ed.; VDE Verlag (Wichmann): Berlin, Germany, 2015; pp. 33-51.

33. Atzl, C.; Scholz, J.; Vockner, B.; Mittlboeck, M.; Knoth, L. Role-Tailored Map Dashboards-A New Approach for Enhancing the Forest-based Supply Chain. ISPRS Int. J. Geo Inf. 2019, 8, 41. [CrossRef]

34. Atzl, C. How to Design Web Maps that Users like. In Online-Karten im Fokus: Praxisorientierte Entwicklung und Umsetzung; Henning, S., Ed.; VDE Verlag (Wichmann): Berlin, Germany, 2015; pp. 33-51.

35. Roth, R.E. Cartographic Interaction Primitives: Framework and Synthesis. Cartogr. J. 2012, 49, $376-395$. [CrossRef]

36. Roth, R.E. An Empirically-Derived Taxonomy of Interaction Primitives for Interactive Cartography and Geovisualization. IEEE Trans. Vis. Comput. Graph. 2013, 19, 2356-2365. [CrossRef] [PubMed]

37. Degbelo, A.; Kauppinen, T. Increasing Transparency through Web Maps. In Proceedings of the Companion Web Conference, Lyon, France, 23-27 April 2018. [CrossRef]

38. Brhel, M.; Meth, H.; Maedche, A.; Werder, K. Exploring principles of user-centered agile software development: A literature review. Inf. Softw. Technol. 2015, 61, 163-181. [CrossRef]

39. Cellina, F.; Bucher, D.; Veiga Simão, J.; Rudel, R.; Martin, R. Beyond Limitations of Current Behaviour Change Apps for Sustainable Mobility: Insights from a User-Centered Design and Evaluation Process. Sustainability 2019, 11, 2281. [CrossRef]

40. Buchenau, M.; Suri, J.F. Experience prototyping. In Proceedings of the 3rd Conference on Designing Interactive Systems: Processes, Practices, Methods, and Techniques, New York, NY, USA, 17-19 August 2000; pp. 424-433.

41. Hartson, R.; Pyla, P.S. The UX Book: Process and Guidelines for Ensuring a Quality User Experience; Elsevier: Amsterdam, The Netherlands, 2012.

42. Lim, Y.K.; Stolterman, E.; Tenenberg, J. The anatomy of prototypes: Prototypes as filters, prototypes as manifestations of design ideas. ACM Trans. Comput. Hum. Interact. (TOCHI) 2008, 15, 1-27. [CrossRef]

43. Beck, K.; Andres, C. Extreme Programming Explained: Embrace Change; Addison-Wesley Professional: Boston, MA, USA, 2000.

44. Hassenzahl, M.; Burmester, M.; Koller, F. AttrakDiff: Ein Fragebogen zur Messung wahrgenommener hedonischer und pragmatischer Qualität. In Mensch \& Computer 2003; Springer: Berlin/Heidelberg, Germany, 2003; pp. 187-196.

45. Hassenzahl, M.; Burmester, M.; Koller, F. Der User Experience (UX) auf der Spur. Zum Einsatz von www.attrakdiff.de. In Usability Professionals 2008; Brau, H., Diefenbach, S., Hassenzahl, M., Koller, F., Peissner, M., Röse Hrsg, K., Eds.; Fraunhofer Verlag: Stuttgart, Germany, 2008. 
46. Leaflet-A JavaScript Library for Interactive Maps. Available online: https:/ /leafletjs.com/ (accessed on 27 July 2020).

47. PostGIS—Spatial and Geographic Objects for PostgreSQL. Available online: https://postgis.net/ (accessed on 27 July 2020). 